\title{
Analysis of Histologic Features Suspecting Anaplastic Lymphoma Kinase (ALK)-Expressing Pulmonary Adenocarcinoma
}

In Ho Choi · Dong Won Kim Sang Yun $\mathrm{Ha}^{1}$. Yoon-La Choi ${ }^{1}$ Hee Jeong Lee ${ }^{2} \cdot$ Joungho Han ${ }^{1}$

Department of Pathology,

Soonchunhyang University Seoul Hospital, Soonchunhyang University College of Medicine, Seoul; 'Department of Pathology, Samsung Medical Center, Sungkyunkwan University School of Medicine, Seoul; ' ${ }^{2}$ epartment of Pathology, Gwangmyeong Sungae Hospital, Gwangmyeong, Korea

Received: March 3, 2015

Revised: May 10, 2015

Accepted: May 12, 2015

Corresponding Author Joungho Han, MD, PhD

Department of Pathology, Samsung Medical Center, Sungkyunkwan University School of Medicine,

81 Irwon-ro, Gangnam-gu, Seoul 135-710, Korea

Tel: $+82-2-3410-2800$

Fax: +82-2-3410-0025

E-mail: haniho@skku.edu

\begin{abstract}
Background: Since 2007 when anaplastic lymphoma kinase (ALK) rearrangements were discovered in non-small cell lung cancer, the $A L K$ gene has received attention due to $A L K$-targeted therapy, and a notable treatment advantage has been observed in patients harboring the EML4/ ALK translocation. However, using ALK-fluorescence in situ hybridization (FISH) as the standard method has demerits such as high cost, a time-consuming process, dependency on interpretation skill, and tissue preparation. We analyzed the histologic findings which could complement the limitation of ALK-FISH test for pulmonary adenocarcinoma. Methods: Two hundred five cases of ALK-positive and 101 of ALK-negative pulmonary adenocarcinoma from January 2007 to May 2013 were enrolled in this study. The histologic findings and ALK immunohistochemistry results were reviewed and compared with the results of ALK-FISH and EGFR/KRAS mutation status. Results: Acinar, cribriform, and solid growth patterns, extracellular and intracellular mucin production, and presence of signet-ring-cell element, and psammoma body were significantly more often present in ALK-positive cancer. In addition, the presence of goblet cell-like cells and presence of nuclear inclusion and groove resembling papillary thyroid carcinoma were common in the ALK-positive group. Conclusions: The above histologic parameters can be helpful in predicting ALK rearranged pulmonary adenocarcinoma, leading to rapid FISH analysis and timely treatment.
\end{abstract}

Key Words: Lung; Adenocarcinoma; Anaplastic large cell lymphoma kinase; Anaplastic lymphoma kinase; In situ hybridization, fluorescence
Two major genetic mutations in non-small cell lung carcinomas (NSCLCs), epidermal growth factor receptor (EGFR) and $K R A S$, have been well studied. Since 2007 when Soda et al. ${ }^{1}$ discovered rearrangements of anaplastic lymphoma kinase (ALK) in NSCLC, the $A L K$ gene has received attention as being responsible for another molecular subtype of lung cancer, accounting for about $3 \%$ to $6 \%$ of NSCLCs. ${ }^{1,2}$ It is important that the presence of $A L K$ gene rearrangement can be an indication for targeted therapy, like EGFR-tyrosine kinase inhibitor (ex. gefitinib) as first-line therapy in patients with advanced pulmonary adenocarcinoma. Moreover, studies have shown that the ALK-targeted inhibitor 'crizotinib' produces a notable positive effect in patients harboring the EML4/ALK translocation. ${ }^{3}$

For the most effective use of $A L K$ inhibitor, an accurate method to detect $A L K$ gene rearrangement should be performed. To date, fluorescence in situ hybridization (FISH) is the universally accepted standard method for detecting $A L K$ rearrangement, with immunohistochemistry (IHC) used as a screening method for identifying $A L K$ rearrangement. ${ }^{4,5}$ However, there is a need for a more rapid method to detect $A L K$ rearrangement due to the highly time-consuming nature of FISH. We encountered a patient who showed a dramatic response to crizotinib treatment before confirming ALK rearrangement by FISH based only IHCpositive results for ALK and suspicious histological findings from a biopsy. Therefore, we were curious whether the combination of IHC and suspicious histological findings can improve upon the shortcomings of FISH. Additionally, limited specimen obtained by biopsy is common and often results in lack of remaining tissue for $A L K$ study after the two major mutation tests (EGFR and $K R A S$ ) for lung cancer. If the histologic finding is more suggestive of $A L K$ mutation, ${ }^{6}$ priority can be given to the $A L K$ mutation test because EGFR, KRAS, and $A L K$ are known to be virtually mutually exclusive.

Our study aimed to investigate the characteristic histologic 
features of ALK-positive pulmonary adenocarcinoma, in order to identify important histologic findings in small biopsied specimens whose architecture is difficult to assess and to evaluate whether the combination of these morphologic features and IHC results can overcome the shortcomings of FISH.

\section{MATERIALS AND METHODS}

\section{Case selection}

Two hundred five ALK-positive cases identified using IHC or FISH on biopsied and resected specimens from Samsung Medical Center were enrolled from January 2007 to May 2013. The basic characteristics of these cases (age, sex, and tumor stage), method of sampling (biopsy or operation), and results of IHC and FISH were investigated.

As a control group for comparing the histologic findings, all consecutive cases of pulmonary resection performed during 2012 were collected. The ALK-IHC had been performed in all cases of the control group, regardless of EGFR and KRAS analyses or microscopic findings.

The Institutional Review Board of Samsung Medical Center approved this study (IRB No. 2014-01-146).

\section{Review of the histological findings}

All resected tumors were classified according to histologic subtype based on the new International Association for the Study of Lung Cancer (IASLC)/American Thoracic Society (ATS)/European Respiratory Society (ERS) classification; the predominant pattern was determined as lepidic, acinar, papillary, solid, and invasive mucinous. All other components ( $>5 \%$ of tumors) were also noted, including the cribriform growth pattern. Presence of extracellular and intracellular mucin, presence of signetring-cells, abrupt presence of goblet cell-like cells that contain amphophilic mucin like those in the intestinal mucosa, and presence of psammoma body, nuclear inclusion and groove, bizarre nuclei and multilobated nuclei were evaluated. As one of the other cytomorphological parameters, presence of prominent large eosinophilic nucleoli was investigated using a cut-off value of $>30 \%$ for tumor cells. In the biopsied specimens, we did not determine the predominant subtype, but we recorded all identifiable growth patterns, nuclear features and presence of psammoma body, bizarre nuclei, and nuclear inclusion and groove. We applied the parameter of 'nuclear inclusion and groove' to cells showing cytomorphologic features of thyroid papillary carcinoma. All cytomorphological features in all presented growth components were evaluated in three fields of each growth pattern us- ing high magnification $(\times 400)$.

\section{$\mathrm{IHC}$ and scoring for ALK}

IHC for $A L K$ fusion was performed using formalin-fixed paraffin-embedded (FFPE) tumor tissue (4- $\mu$ m thickness) and an antibody to NCL-ALK (1:30, clone 5A4, Novocastra, Newcastle upon Tyne, UK). The interpretation of IHC results was based on the a four-tier scoring system: 0 (none), $1+$ (faint cytoplasmic staining, $\geq 10 \%$ of tumor cells), $2+$ (moderate, smooth cytoplasmic staining), and $3+$ (intense, granular cytoplasmic staining) IHC scores of $2+$ or $3+$ were regarded as ALK-positive results. ${ }^{5}$

\section{FISH of ALK}

The FISH analysis for $A L K$ rearrangement on FFPE tissue was examined using a probe specific to the $A L K$ locus (Vysis LSI ALK dual-color, Break-Apart Rearrangement Probe, Abbott Molecular, Abbott Park, IL, USA). At least 50 nonoverlapping tumor cells were examined, and the cut-off value for positive $A L K$ rearrangement was defined as $>15 \%$ of tumor cells showing split signals or lone 3 ' (orange color) signals (Fig. 1).

\section{EGFR and KRAS mutation status}

EGFR and KRAS mutation data of $A L K$-negative and $A L K$ positive groups were investigated. The mutation analyses of EGFR (exon 18, 19, 20, and 21) and KRAS (exons 2 and 3) were examined using direct sequencing-polymerase chain reaction

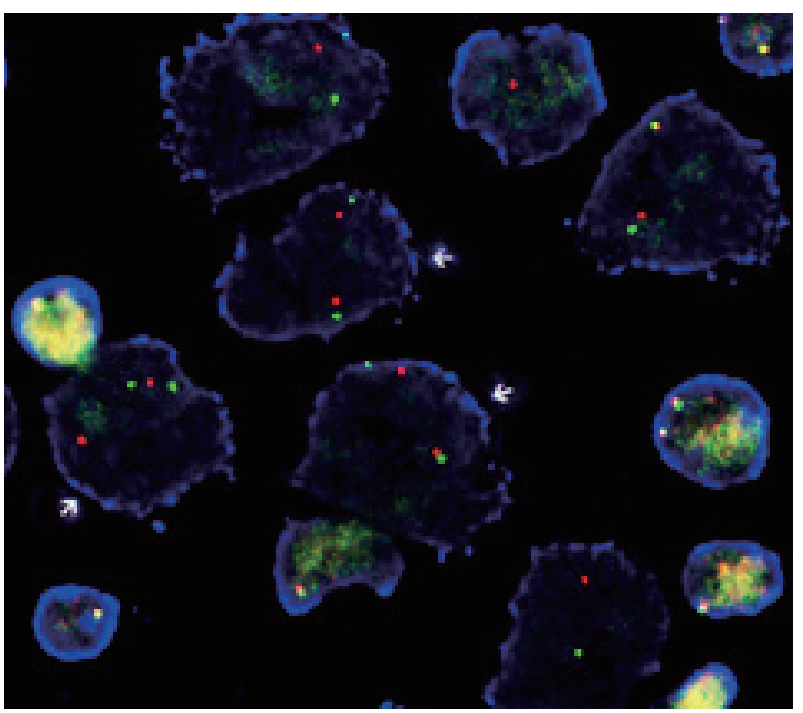

Fig. 1. Fluorescence in situ hybridization (FISH) of anaplastic lymphoma kinase (ALK)-rearranged pulmonary adenocarcinoma. Rearranged tumor nuclei show split signals (arrows) of $3^{\prime}$ (orange color) and $5^{\prime}$ (green color) ends of the gene hybridized using a dualcolor ALK break-apart FISH probe (Vysis LSI ALK Dual Color, Break Apart Rearrangement Probe, Abbott Molecular). 
(PCR). Genomic DNA was extracted from the FFPE tissue and purified with a QIAquick PCR purification kit (Qiagen, Hilden, Germany). Bidirectional sequencing was performed using the BigDye Terminator v 1.1 kit (Applied Biosystems, Foster City, CA, USA) on an ABI 3130xl genetic analyzer (Applied Biosystems).

\section{Statistical analysis}

Fisher exact test was used to compare the features of ALK-positive pulmonary adenocarcinoma according to sex, and linear by linear association was used for age and tumor stage. Normal distribution according to age in each group was tested by Kolmogorove-Smirnove and Shapiro-Wilk test. Chi-square test/Fisher exact test, independent samples $t$ test, and logistic regression analysis were applied where appropriate in order to determine the relationship between morphological features and ALK positivity. A p-value of $<.05$ was considered to be statistically significant in all statistical tests. All analyses were performed using SPSS ver.
18.0 (SPSS Inc., Chicago, IL, USA).

\section{RESULTS}

\section{Comparison of ALK-IHC and ALK-FISH}

The status of ALK-FISH and ALK-IHC was compared in 78 cases (Table 1). Among 69 ALK-FISH positive cases, 63 cases showed $2+$ or $3+$ positivity in ALK-IHC staining; therefore, the sensitivity of ALK-IHC $(2+$ and $3+)$ was determined to be $91.3 \%(63 / 69)$.

\section{Characteristics of patients with ALK-positive pulmonary adenocarcinoma}

We settled ALK-positive group ( $\mathrm{n}=205)$ as ALK-IHC or ALK-FISH positive cases, including 127 cases of ALK-IHC positive (2+ or 3+) without ALK-FISH examination, 63 cases of positive ALK-IHC and ALK-FISH, 9 cases of positive ALKIHC and not detected or failed ALK-FISH, and 6 cases of nega-

Table 1. Arrangement of ALK-FISH results according to ALK-IHC and sampling method

\begin{tabular}{|c|c|c|c|c|}
\hline & \multicolumn{3}{|c|}{ ALK-FISH } & \multirow{2}{*}{ No. of cases $(n=78)$} \\
\hline & Rearranged $(n=69)$ & Not detected $(n=6)$ & Failed $(n=3)$ & \\
\hline \multicolumn{5}{|l|}{ ALK-IHC } \\
\hline Negative & $1(1.4)$ & 0 & 0 & 1 \\
\hline $1+$ & $3(4.3)$ & 0 & 0 & 3 \\
\hline $2+$ & $42(60.9)$ & $3(50.0)$ & $3(100)$ & 48 \\
\hline $3+$ & $21(30.4)$ & $3(50.0)$ & 0 & 24 \\
\hline Failed & $2(2.9)$ & 0 & 0 & 2 \\
\hline \multicolumn{5}{|c|}{ Sampling method } \\
\hline Biopsy & $56(81.2)$ & $2(33.3)$ & 0 & 58 \\
\hline Resection & $13(18.8)$ & $4(66.7)$ & $3(100)$ & 20 \\
\hline
\end{tabular}

Values are presented as number (\%).

ALK, anaplastic lymphoma kinase; FISH, fluorescence in situ hybridization; IHC, immunohistochemistry.

Table 2. Characteristics of patients with ALK-positive and -negative pulmonary adenocarcinoma

\begin{tabular}{|c|c|c|c|c|c|c|}
\hline \multirow{2}{*}{\multicolumn{2}{|c|}{ Total cases $(n=306)$}} & \multicolumn{3}{|c|}{ ALK-positive } & \multirow{2}{*}{$\frac{\text { ALK-negative }}{\text { Resection }(n=101)}$} & \multirow{2}{*}{$p$-value } \\
\hline & & Biopsy $(n=129)$ & Resection $(n=76)$ & Total $(n=205)$ & & \\
\hline \multirow{2}{*}{\multicolumn{2}{|c|}{ Sex (M:F) }} & $47: 82$ & $36: 40$ & $83: 122$ & $53: 48$ & $.047^{a}$ \\
\hline & & & & & & $.501^{b}$ \\
\hline \multirow{2}{*}{\multicolumn{2}{|c|}{ Age (yr) }} & $53.9 \pm 11.5$ & $55.1 \pm 11.7$ & $54.4 \pm 11.5$ & $61.7 \pm 8.8$ & $<.001^{\mathrm{a}}$ \\
\hline & & & & & & $<.001^{\mathrm{b}, \mathrm{c}}$ \\
\hline \multirow[t]{7}{*}{ TNM stage, n (\%) } & a & $1(0.8)$ & 29 (38.2) & 30 (14.6) & 20 (19.8) & $.234^{\mathrm{b}, \mathrm{d}}$ \\
\hline & $1 b$ & $1(0.8)$ & $4(5.3)$ & $5(2.4)$ & $14(13.9)$ & \\
\hline & $2 a$ & $4(3.1)$ & $8(10.5)$ & $12(5.9)$ & $12(11.9)$ & \\
\hline & $2 b$ & $1(0.8)$ & 0 & $1(0.5)$ & $8(7.9)$ & \\
\hline & $3 a$ & $7(5.4)$ & 23 (30.3) & 30 (14.6) & $32(31.7)$ & \\
\hline & $3 b$ & $9(7.0)$ & $3(3.9)$ & $12(5.9)$ & $1(1.0)$ & \\
\hline & 4 & 106 (82.2) & $9(11.8)$ & 115 (56.1) & 14 (13.9) & \\
\hline
\end{tabular}

ALK, anaplastic lymphoma kinase; M, male; F, female.

${ }^{a} p$-value between the ALK-positive and -negative group; ${ }^{b} p$-value between the ALK-positive and -negative resected group; ${ }^{c} p$-value by Student's t test; ${ }^{d L i n e a r}$ by linear association by chi-square test. 
tive $(0$ or $1+)$ or failed ALK-IHC and positive ALK-FISH.

The characteristics of patients in the ALK-positive group and control group are presented in Table 2. The ALK-positive group consisted of 129 biopsy cases and 76 resection cases. The sex ratio in the ALK-positive group was male:female = 83:122 in contrast to 53:48 in the ALK-negative group. This finding was statistically significant $(\mathrm{p}=.047)$ and suggested that ALK-positive pulmonary adenocarcinoma occurs more frequently in women, with odds ratio of 1.623. ALK-positive cancer occurred in patients 7.3 years younger than the ALK-negative group, and this difference was statistically significant $(\mathrm{p}<.001)$. The distribution of tumor stage in the ALK-positive biopsy group was one-sided in stage $4(82.2 \%)$. However, a statistically significant difference was not noted between the ALK-positive and -negative resection groups $(\mathrm{p}=.234)$.
Histological type and characteristic microscopic findings in ALK-positive pulmonary adenocarcinoma

Histologic subtypes based on predominant growth pattern were investigated in the ALK-positive and -negative resection groups, but there was no statistically significant histologic subtype of ALK-positive pulmonary adenocarcinoma (Table 3). However, acinar, solid, micropapillary, and cribriform growth patterns were more frequent in ALK-positive lung cancer based on the examination of growth patterns $(\mathrm{p}=.035, \mathrm{p}=.002, \mathrm{p}=.004$, and $\mathrm{p}<.001$ respectively) (Table 3, Fig. 2A, B). The ALK-positive group showed more variable growth patterns at a statistically significant level; $77.7 \%$ of cases in the ALK-positive group showed more than 3 types of growth patterns (mean, 3.3). However, cases in the ALK-negative group usually contained 2 or 3 types of growth patterns $(75.2 \%$; mean, 2.4) $(\mathrm{p}<.001)$ (Table 3, Fig. 2A).

Table 3. Correlation of histologic findings in resection groups according to ALK positivity

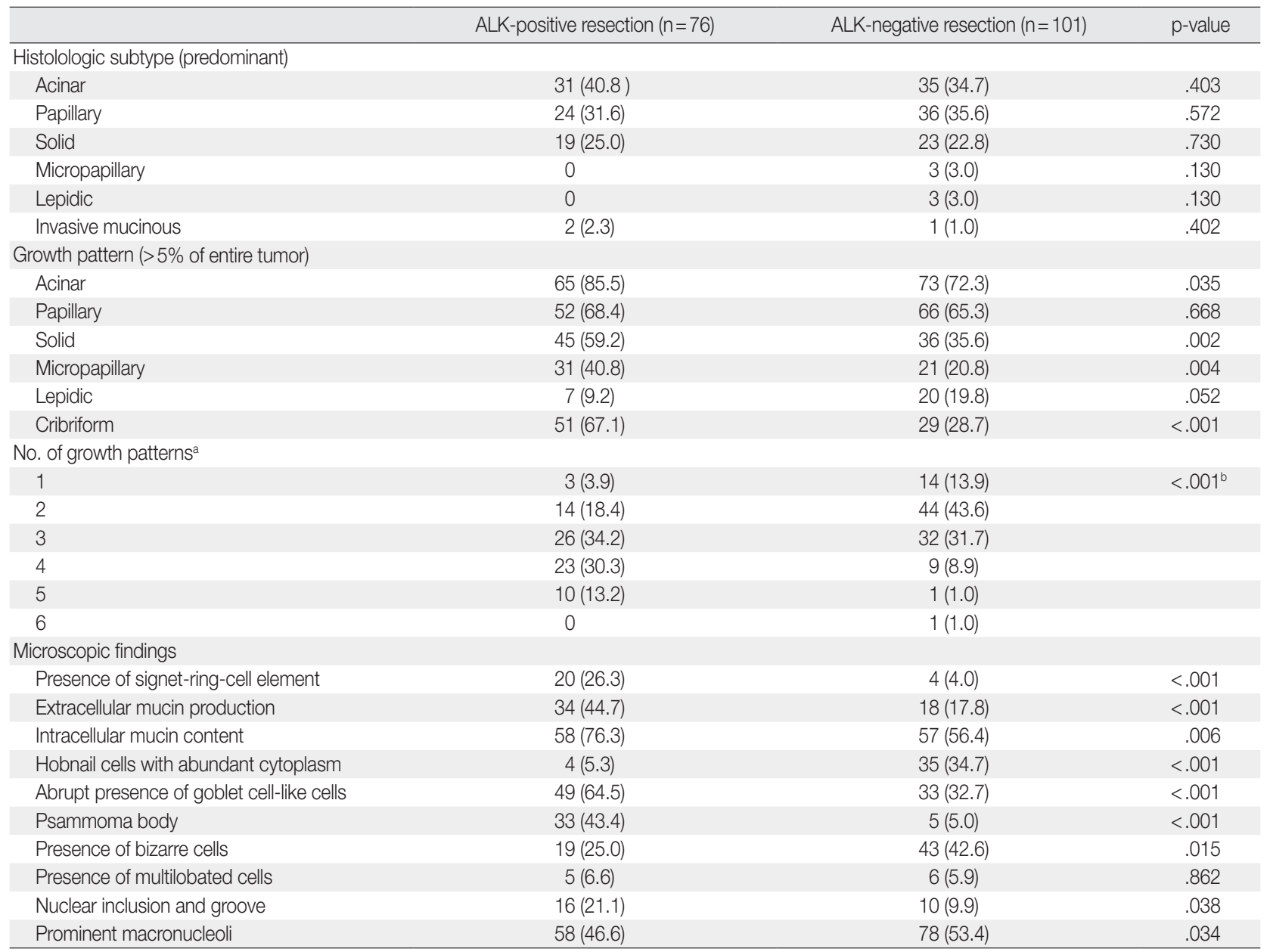

Values are presented as number (\%).

ALK, anaplastic lymphoma kinase.

aNumber of all presented growth patterns presented in 'Growth pattern (> $5 \%$ of entire tumor)'; ' $p$-value by Student's t-test. 
Among the examined cytomorphologic parameters, extracellular and intracellular mucin production (Fig. 2C), presence of signet-ring-cell element (Fig. 2D), presence of psammoma body (Fig. 2E), abrupt presence of goblet cell-like cells (Fig. 2F), and presence of nuclear inclusion and groove (Fig. 2G) were significantly more frequent in the ALK-positive resection group. Hobnail cells with abundant cytoplasm, bizarre cells, and presence of macronucleoli were more frequently observed in the ALK-neg-
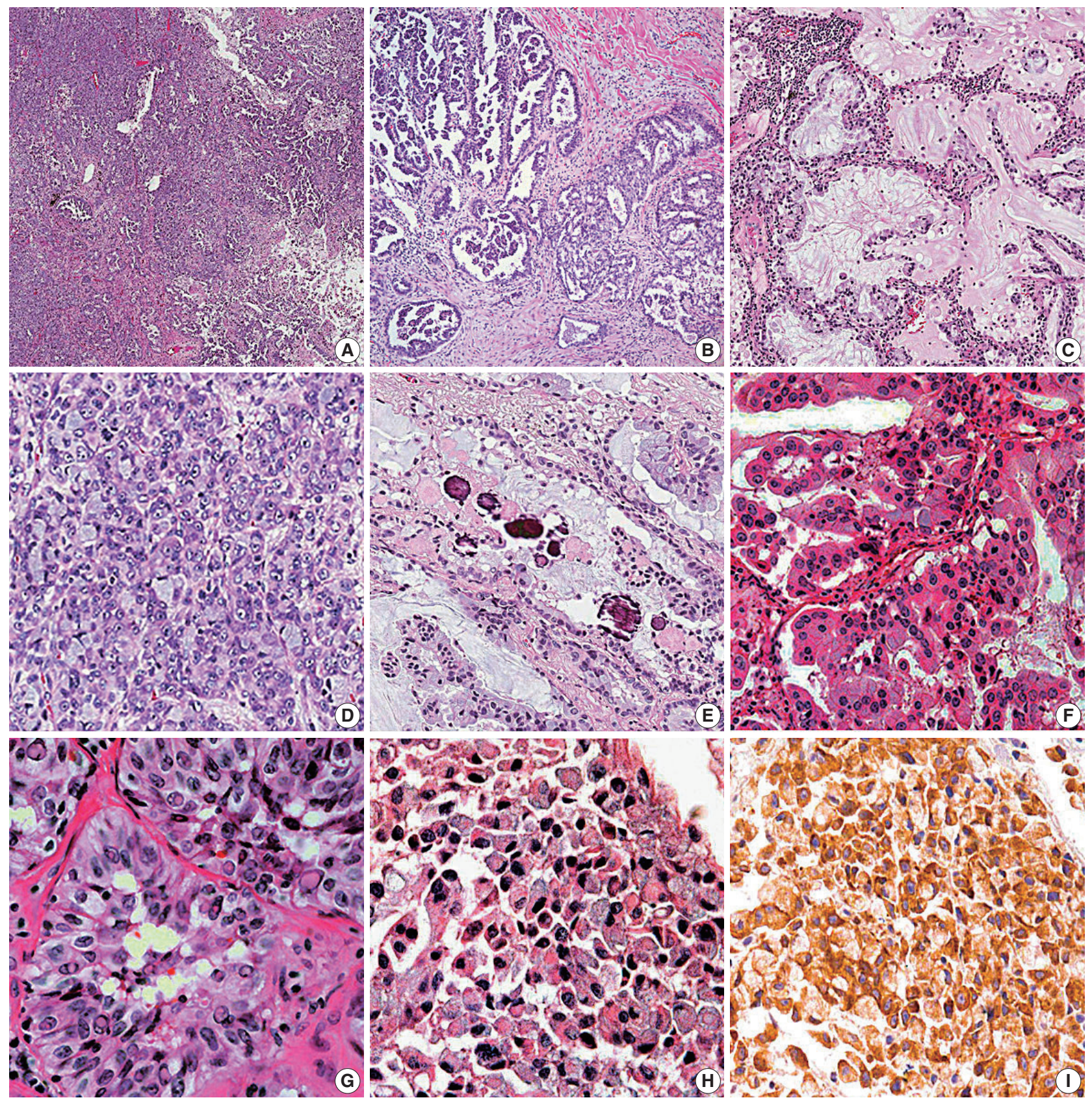

Fig. 2. Histologic features of anaplastic lymphoma kinase (ALK)-rearranged pulmonary adenocarcinoma. (A) ALK-rearranged tumors show variable growth patterns. At low magnification, the tumor shows solid, acinar, and papillary growth patterns. (B) Cribriform and micropapillary patterns are more frequent in ALK-positive pulmonary adenocarcinoma. (C) Tumors have frequent extracellular and intracellular mucin production. (D) Solid growth is frequently found to contain signet-ring-cells. (E) A few psammoma bodies are noted in the background of mixed tumor and extracellular mucin. (F) Some tumors contain goblet cell-like cells. (G) Intranuclear inclusion and nuclear groove resembling papillary thyroid carcinoma are frequently found. $(H)$ On the biopsied specimen from the patient showing dramatic response to $A L K$-targeted therapy before ALK-fluorescence in situ hybridization, many signet-ring-cells are found. (I) Tumor cells in Fig. 2H show 3+ ALK-immunohistochemistry score. 
Table 4. Multivariate analysis: histologic findings in ALK-positive or -negative resection groups

\begin{tabular}{|c|c|c|c|c|}
\hline & ALK-positive resection $(n=76)$ & ALK-negative resection $(n=101)$ & Odds ratio & $95 \% \mathrm{Cl}$ \\
\hline \multicolumn{5}{|l|}{ Growth pattern (>5\% of entire tumor) } \\
\hline Acinar & $65(85.5)$ & $73(72.3)$ & 3.178 & $1.298-7.779$ \\
\hline Solid & $45(59.2)$ & $36(35.6)$ & 2.694 & $1.301-5.577$ \\
\hline Micropapillary & $31(40.8)$ & $21(20.8)$ & 3.414 & $1.581-7.372$ \\
\hline Cribriform & $51(67.1)$ & $29(28.7)$ & 3.771 & $1.877-7.575$ \\
\hline \multicolumn{5}{|l|}{ Microscopic findings } \\
\hline Presence of signet-ring-cell element & $20(26.3)$ & $4(4.0)$ & 5.466 & $1.488-20.080$ \\
\hline Extracellular mucin production & $34(44.7)$ & $18(17.8)$ & 3.372 & $1.411-8.057$ \\
\hline Hobnail cells with abundant cytoplasm & $4(5.3)$ & $35(34.7)$ & 0.096 & $0.026-0.356$ \\
\hline Psammoma body & $33(43.4)$ & $5(5.0)$ & 16.548 & $5.303-51.641$ \\
\hline
\end{tabular}

Values are presented as number (\%).

ALK, anaplastic lymphoma kinase; $\mathrm{Cl}$, confidence interval.

Table 5. Correlation of morphologic features between ALK-positive biopsy and resection groups

\begin{tabular}{lccr}
\hline & ALK-positive biopsy $(\mathrm{n}=129)$ & ALK-positive resection $(\mathrm{n}=76)$ & $\mathrm{p}$-value \\
\hline Presence of signet-ring-cell element & $40(31.0)$ & $20(26.3)$ & .476 \\
Extracellular mucin production & $15(11.6)$ & $34(44.7)$ & $<.001$ \\
Intracellular mucin content & $101(78.3)$ & $58(76.3)$ & .743 \\
Hobnail cells with abundant cytoplasm & $5(3.9)$ & $4(5.3)$ & .640 \\
Abrupt presence of goblet cell-like cells & $69(53.5)$ & $49(64.5)$ & .124 \\
Psammoma body & $20(15.5)$ & $33(43.4)$ & $<.001$ \\
Nuclear inclusion and groove & $7(5.4)$ & $16(21.1)$ & .001 \\
\hline
\end{tabular}

Values are presented as number (\%).

ALK, anaplastic lymphoma kinase.

ative group.

According to logistic regression analysis (Table 4), acinar, solid, micropapillary, and cribriform growth patterns were more frequent in ALK-positive group, and the significant cytomorphologic features included presence of signet-ring-cell elements, extracellular mucin production, hobnail cells with abundant cytoplasm, and psammoma body.

\section{Morphological features of small biopsy specimens compared with resection specimens}

To evaluate whether ALK-specific morphologic features in the resection group can be applied to small biopsy specimens, cytomorphologic findings were compared between the ALK-positive biopsy and resection groups (Table 5). The parameters except for extracellular mucin production, psammoma body, and nuclear inclusion and groove showed no differences between the biopsy and resection groups ( $\mathrm{p}>.05)$.

\section{DISCUSSION}

Since the presence of $A L K$ rearrangement has become an important predictive biomarker for the use of the $A L K$-targeted inhibitor 'crizotinib' for NSCLC, the detection of $A L K$ rearrange- ments is also becoming increasingly important. However, ALK$\mathrm{FISH}$ as a standard method for detecting $A L K$ rearrangement is expensive, time-consuming, and influenced by many factors (skill and material). On the other hand, ALK-IHC has been used as a screening method due to its lower specificity compared to ALKFISH. We evaluated the histologic findings that can be helpful in predicting $A L K$ rearrangement and in saving time until $A L K$ targeted inhibitor treatment.

Although there might have been a selection bias due to the excessive number of biopsied cases in stage IV, the ALK-positive group showed more frequent occurrence of lung adenocarcinoma in an advanced tumor stage than did the ALK-negative group (stage IV: $56.1 \%$ vs $13.9 \%$ ). Although the ALK-positive resection group showed no statistically significant difference in the distribution of tumor stage compared with the ALK-negative group ( $\mathrm{p}=.234)$, ALK-positive lung cancer is suspected to show more aggressive behavior, considering that ALK-positive lung cancer presents in an advanced stage with low resectability, leading to the need for chemotherapy or palliative therapy instead of surgical treatment.

There have been several studies on the morphologic findings of $A L K$-rearranged NSCLCs. Some authors ${ }^{7-9}$ have suggested that acinar growth pattern and extracellular mucin production 
are typical of ALK-rearranged pulmonary adenocarcinoma. Another author recommended signet-ring-cell elements associated with solid growth as a feature of $A L K$-rearranged pulmonary adenocarcinoma. ${ }^{10}$ In the present study, histologic subtype based on 'predominant' growth pattern was not effective in discriminating between the ALK-positive and -negative groups. However, ALKpositive pulmonary adenocarcinoma showed more variable growth patterns (Fig. 2A) with significantly more frequent presence of acinar, solid, micropapillary, and cribriform growth patterns (Fig. 2A, B, D) compared with non- $A L K$ rearranged adenocarcinoma (Table 3).

As reported by Jokoji et al. ${ }^{7}$ and Inamura et al., ${ }^{9}$ who observed that mucin production was one of the important findings of EML4-ALK-positive lung adenocarcinoma, our study also showed that extracellular mucin production was statistically significant in the ALK-positive resection group ( $\mathrm{p}<.001$ ).

Rodig et al..$^{10}$ showed that tumor cells forming a solid or sheetlike pattern were easily distinguishable from other growth patterns in $A L K$-rearranged adenocarcinoma, and that the majority (56\%) of $A L K$-rearranged tumors showed a solid growth pattern with $>10 \%$ signet-ring cells. Our study also showed frequent solid growth (45/76 cases, 59.2\%) in the ALK-positive resection group, and signet-ring-cell elements were extremely frequent in the ALK-positive resection group (20/76 cases, $26.3 \%$ vs $4 / 101$ cases, 4.0\%) (Table 3, Fig. 2D). Indeed, the signet-ring-cell element was significantly more frequent in the ALK-positive resection group showing solid growth than in the ALK-negative group (odds ratio, 21.9 vs 1.9).

A recent study about histomorphologic features of $A L K$-rearranged lung adenocarcinoma by Kim et al. ${ }^{11}$ showed several significant microscopic features, including cribriform formation, presence of extracellular mucin, presence of mucin-containing cells, close relation to adjacent bronchioles, presence of psammoma body, presence of cholesterol cleft, and solid predominant pattern. Their data are similar to those of the present study except for two parameters that we did not evaluate close relation to adjacent bronchioles and presence of cholesterol cleft. However, we are more interested in the abrupt presence of goblet cell-like cells and presence of nuclear inclusion and groove resembling papillary thyroid carcinoma.

Abrupt presence of goblet cell-like cells (Fig. 2F) was frequent in the ALK-positive resection group (64.5\% vs $32.7 \%, \mathrm{p}<.001$ ). We also found that many cases with a signet-ring-cell element in the ALK-positive resection group showed abrupt presence of goblet cell-like cells (16/20 cases, 80.0\%). Considering the cytomorphologic similarity between signet-ring cells and goblet cell- like cells, we supposed that presence of goblet cell-like cells is part of a process to form signet-ring-cell and is predictive parameter for ALK-positive pulmonary adenocarcinoma. However, this relation was not statistically significant $(\mathrm{p}=.091)$.

One of the notable findings of our study was that the presence of nuclear inclusion and groove resembling papillary thyroid carcinoma (Fig. 2G) was noted more often observed in the ALKpositive lung cancer cases compared to the negative cases. We hypothesized that this feature is valuable in predicting ALKpositive lung cancer. However, there is no available data on why papillary thyroid carcinoma-like features are more frequent in ALK-positive lung cancer, suggesting additional study cases are needed.

On the correlation between biopsy and resection cases in ALK-positive tumors (Table 5), we found that some parameters, such as presence of signet-ring-cell elements, intracellular mucin content, and presence of goblet cell-like cells, showed no statistically significant differences between the biopsy and resection groups. However, these features are believed to be helpful for predicting ALK positivity in small biopsy specimens. Furthermore, presence of signet-ring-cell, psammoma body, nuclear inclusion and groove, and goblet cell-like cells are meaningful in small biopsy sections, if present (Fig. 2H).

Paik et $a l^{4}$ reported that the sensitivity and specificity of ALKIHC was $100 \%$ and $95.8 \%$, respectively, with a well-organized interpretation flow and concluded that ALK-IHC might be useful as a screening method to identify $A L K$ rearrangements; a 91.3\% sensitivity of ALK-IHC was noted in our study. However, ALK-IHC alone is not yet adequate to replace ALK-FISH and is applicable only as a screening method. For example, we excluded 4 cases showing discordance between the result of $A L K$ and that of EGFR and KRAS before the present study. These cases included three EGFR-mutated cases (two ALK-IHC- and $E G F R$-positive cases with no examination by ALK-FISH and one concurrent EGFR-mutated and ALK-FISH-positive case) and one concurrent ALK-IHC- and KRAS-positive case without examination by ALK-FISH; KRAS missense mutation in the 12th codon : c.34G > T (p.G12C). Upon reviewing a case showing EGFR missense mutation in exon 18 (G719X), ALK-IHCpositivity (2+), and ALK-FISH-positivity, there was a suspicion of over-interpretation of ALK-FISH analysis; about $20 \%$ of tumor cells showed only 'lone 3' (orange color) signals' without 'split signals.' The reasons for the other three false positive cases by ALK-IHC could not be determined, although they showed definite $2+$ and $3+$ ALK-IHC scoring.

However, ALK-FISH has some demerits, such as high cost, 
time-consuming process, dependency on many conditions, including skill of interpretation, and preparation of material such as quantity and quality of tumor cells. In our study, 9 cases showed no detection or failed results on ALK-FISH, and they were all negative for EGFR and KRAS, despite having 2+ or 3+ ALKIHC scores (Table 1). After review of ALK-IHC and ALK-FISH results, the 6 cases with no detection were also confirmed as failures due to interpretation difficulty of ALK-FISH caused by excessive mucin content and low cellularity. In the practice, ALKFISH is usually the second choice after analyses of both major mutations (EGFR and KRAS), and it leads to scarcity of tissue availability for testing from small biopsied specimens. Therefore, we believe that ALK-FISH could be complemented for rapid treatment, and the microscopic findings might be predictors of $A L K$ rearrangement. If the morphologic findings are suggestive of $A L K$-rearranged pulmonary adenocarcinoma and are supported by a positive result in ALK-IHC, the patients can benefit from timely treatment. For example, we encountered a patient who showed dramatic response to 'crizotinib' before confirmation by ALK-FISH using only positive ALK-IHC and suspicious histological findings via biopsy (Fig. 2H, I).

Conclusively, we found that acinar, micropapillary, cribriform, and solid growth patterns with intracellular mucin content, signet-ring-cell element, and psammoma body were useful in predicting $A L K$ rearrangement. Indeed, the notable findings of our study were that presence of goblet cell-like cells or nuclear inclusion or groove resembling papillary thyroid carcinoma was more common in ALK-positive adenocarcinoma of the lung, although these findings should be evaluated further. In the biopsied specimens, presence of signet-ring-cell elements, goblet cell-like cells, intracellular mucin content, and psammoma body can be helpful in predicting $A L K$ rearrangement. Additionally, there is a limitation of our study as the histologic comparison between FISHpositive cases and IHC-positive cases could not be made due to weighted biopsied specimens.

We hope to clarify the histologic findings of $A L K$ rearrangement in a prospective cohort study and to evaluate the histologic findings with newly appearing genes in $A L K$ rearrangement.

\section{Conflicts of Interest}

No potential conflict of interest relevant to this article was reported.

\section{REFERENCES}

1. Soda M, Choi YL, Enomoto M, et al. Identification of the transforming EML4-ALK fusion gene in non-small-cell lung cancer. Nature 2007; 448: 561-6.

2. Sun JM, Lira M, Pandya K, et al. Clinical characteristics associated with $A L K$ rearrangements in never-smokers with pulmonary adenocarcinoma. Lung Cancer 2014; 83: 259-64.

3. O’Bryant CL, Wenger SD, Kim M, Thompson LA. Crizotinib: a new treatment option for ALK-positive non-small cell lung cancer. Ann Pharmacother 2013; 47: 189-97.

4. Paik JH, Choe G, Kim H, et al. Screening of anaplastic lymphoma kinase rearrangement by immunohistochemistry in non-small cell lung cancer: correlation with fluorescence in situ hybridization. J Thorac Oncol 2011; 6: 466-72.

5. Yi ES, Boland JM, Maleszewski JJ, et al. Correlation of IHC and FISH for ALK gene rearrangement in non-small cell lung carcinoma: IHC score algorithm for FISH. J Thorac Oncol 2011; 6: 459-65.

6. Yoshida A, Tsuta K, Nakamura H, et al. Comprehensive histologic analysis of $A L K$-rearranged lung carcinomas. Am J Surg Pathol 2011; 35: 1226-34.

7. Jokoji R, Yamasaki T, Minami S, et al. Combination of morphological feature analysis and immunohistochemistry is useful for screening of EML4-ALK-positive lung adenocarcinoma. J Clin Pathol 2010; 63: 1066-70.

8. Inamura K, Takeuchi K, Togashi Y, et al. EML4-ALK fusion is linked to histological characteristics in a subset of lung cancers. J Thorac Oncol 2008; 3: 13-7.

9. Inamura K, Takeuchi K, Togashi Y, et al. EML4-ALK lung cancers are characterized by rare other mutations, a TTF-1 cell lineage, an acinar histology, and young onset. Mod Pathol 2009; 22: 508-15.

10. Rodig SJ, Mino-Kenudson M, Dacic S, et al. Unique clinicopathologic features characterize $A L K$-rearranged lung adenocarcinoma in the western population. Clin Cancer Res 2009; 15: 5216-23.

11. Kim H, Jang SJ, Chung DH, et al. A comprehensive comparative analysis of the histomorphological features of $A L K$-rearranged lung adenocarcinoma based on driver oncogene mutations: frequent expression of epithelial-mesenchymal transition markers than other genotype. PLoS One 2013; 8: e76999. 